\title{
Design and characterisation of a screen-printed millimetre-wave flexible metasurface using copper ink for communication applications
}

To cite this article before publication: Oluyemi P Falade et al 2018 Flex. Print. Electron. in press https://doi.org/10.1088/2058-8585/aaf0eb

\section{Manuscript version: Accepted Manuscript}

Accepted Manuscript is "the version of the article accepted for publication including all changes made as a result of the peer review process, and which may also include the addition to the article by IOP Publishing of a header, an article ID, a cover sheet and/or an 'Accepted

Manuscript' watermark, but excluding any other editing, typesetting or other changes made by IOP Publishing and/or its licensors"

This Accepted Manuscript is @ 2018 IOP Publishing Ltd.

During the embargo period (the 12 month period from the publication of the Version of Record of this article), the Accepted Manuscript is fully protected by copyright and cannot be reused or reposted elsewhere.

As the Version of Record of this article is going to be / has been published on a subscription basis, this Accepted Manuscript is available for reuse under a CC BY-NC-ND 3.0 licence after the 12 month embargo period.

After the embargo period, everyone is permitted to use copy and redistribute this article for non-commercial purposes only, provided that they adhere to all the terms of the licence https://creativecommons.org/licences/by-nc-nd/3.0

Although reasonable endeavours have been taken to obtain all necessary permissions from third parties to include their copyrighted content within this article, their full citation and copyright line may not be present in this Accepted Manuscript version. Before using any content from this article, please refer to the Version of Record on IOPscience once published for full citation and copyright details, as permissions will likely be required. All third party content is fully copyright protected, unless specifically stated otherwise in the figure caption in the Version of Record.

View the article online for updates and enhancements. 


\title{
Design and characterisation of a screen-printed millimetre-wave flexible metasurface using copper ink for communication applications
}

\author{
Oluyemi P Falade*1, Syeda Fizzah Jilani*1, Abshir Yusuf Ahmed ${ }^{1}$, Tom Wildsmith², Paul Reip ${ }^{2}$, \\ Khalid Z. Rajab ${ }^{1}$ and Akram Alomainy ${ }^{1}$ \\ ${ }^{1}$ School of Electronic Engineering and Computer Science, Queen Mary University of London, London E1 4NS, U.K. \\ ${ }^{2}$ Intrinsiq Materials Limited, Farnborough, GU14 0LX, U.K.
}

*These authors contributed equally and to whom any correspondence should be addressed

E-mail: s.f.jilani@qmul.ac.uk and o.p.falade@qmul.ac.uk

Received xxxxxx

Accepted for publication $\mathrm{xxxxxx}$

Published xxxxxx

\section{Abstract}

In this paper, a design, fabrication and characterisation of metasurfaces with the anisotropic response for millimetre waves (MMW) have been presented for the seamless, secure and low-loss high-frequency communication. Two frequency bands, 29 $\mathrm{GHz}$ and $60 \mathrm{GHz}$ have been selected for the development of an efficient, cost-effective and broadband metasurfaces constituting an octa-armed star-shaped and symmetric dual orthogonally I-shaped structures respectively. Low-cost and highperformance nanoscale printing inks and pastes have been deployed in the screen printing technique in order to perform the fabrication of designed metasurfaces on the flexible films. Electromagnetic (EM) characteristics of the proposed metasurfaces are evaluated by using the state-of-the-art testing facilities to demonstrate their potential performance attributes in wavepropagation at MMW for high data rate communication links.

Keywords: 5G, flexible, metasurface, millimetre-wave, wireless

\section{Introduction}

Printed devices and circuits have accomplished significant advancements over the past decades and are regarded as one of the highly progressing sectors in the electronics industry. Printed devices constitute the advantages of low cost, thin form factor, conformity, flexibility, rollability, lightweight and high robustness towards cracks while stretching, bending and folding [1]. These characteristics have a massive impact in wearables, smart packaging, body-centric sensors and many other applications. Several efficient additive manufacturing processes such as, inkjet printing [2], flexography [3], screen printing [4], and additive photolithography such as lift off and electroplating [5] have gained success in the production of printed flexible wireless electronics. These additive processes are usually deployed by a controlled layer-by-layer deposition or growth sequence that ensures accurate prototyping [6, 7]. Among the fast and high-resolution printing techniques, screen printing is found promising due to the capabilities of simplicity, affordability, time-efficiency and reduced material wastage [6]. Screen printing by using conductive pastes and metal epoxies has gained notable attention in printable electronics and PCB industry due to the ability to perform patterning of conductive lines, pads and metallic structures. Inspired by the EM characteristics of the metasurfaces, this paper exploits the idea to utilise a screen-printing technique in realising a flexible MMW indoor communication link.

Metamaterials are synthetic composite materials with a structure that possesses characteristics not commonly found in natural materials such as a negative refractive index, negative permittivity and negative permeability [8-11]. Metamaterials are usually arranged in a set of subwavelength scatters within 
a region of space that generates the required EM properties as bulk materials in a three-dimensional (3D) format. Strong dispersion, bulky nature, high losses, and 3D analogues nature of metamaterials, and complicated fabrication principles have hindered the practical applications of the metamaterials. Currently, metasurface; an equivalent of the metamaterial is being used due to its simplicity, light weight, reduced profile and ease of fabrication that makes it more appealing and easier to realise from a practical point of view. Several processes such as lithography, inkjet printing etc., have been suggested in order to integrate repeated metamaterial structures on different surfaces and photonic chip and are well suited for a mass production [12]. Metasurfaces are two-dimensional artificial materials with thicknesses much smaller than the incident light wavelength, allowing complete control over the phase, amplitude, and polarisation of the EM-wave beams [13-16]. Numerous applications of metasurface such as, in ultrathin planar reflect-array lenses, polarisation-controlled plasmonic coupler, and Photonic Spin Hall Effect etc., make it suitable for transmission and reception of a signal with tuning flexibility in wireless technologies [17-20].

The evolution of $5^{\text {th }}$ generation $(5 \mathrm{G})$ wireless networks have emphasised the need of implementing a new architecture based on MMW spectrum to accomplish the overwhelming capacity demands [21]. In this regard, MMW band at $28 \mathrm{GHz}$ is recommended by the global standardisation bodies due to comparatively lower attenuations and ability to provide multigigabit rates in transmission links for emerging broadband wireless networks [22]. In contrast, $60 \mathrm{GHz}$ band has been anticipated as a suitable choice for well-secured and highspeed point-to-point links for short-range indoor applications due to high attenuation level observed at this bandwidth [23, 24]. Implementation of such an immense transformation from low-frequency spectrum to MMW bands demands much more research efforts in the realisation of efficient MMW based devices, components, antennas and wireless applications. It is also established that the advantages of metasurfaces could be utilised in MMW communication systems. In this research, flexible and low-cost symmetric reflective metasurfaces with a control on $\mathrm{x}$ - and $\mathrm{y}$-polarised reflected waves are designed, fabricated and tested for $29 \mathrm{GHz}$ and $60 \mathrm{GHz}$ communication links. It is worth mentioning that $29 \mathrm{GHz}$ band is merely a representation of the designed resonant dip at this point, however, it interprets an operating range of $26-31 \mathrm{GHz}$ which includes $28 \mathrm{GHz} 5 \mathrm{G}$ band, as well as $26 \mathrm{GHz}$ which is another potential $5 \mathrm{G}$ candidate. The concept is to utilise metasurfaces in the indoor point-to-point links which can be easily applied as wallpaper on the walls without the structural loading concern to existing construction [25]. This would replace the need of installing hardwired solutions (copper/optical fibre) directly into buildings for delivering high-speed broadband.

In this paper, symmetrical octa-armed star-shaped unit-cell designed at $28 \mathrm{GHz}$ band is repeated in a metasurface pattern, while a symmetric metasurface I-shaped orthogonal structure is designed at $60 \mathrm{GHz}$. In order to introduce the conformity and effectiveness in the practical applications, thin flexible films have been used as a substrate material. The scope of screen printing in the development of high-frequency printed metasurfaces is investigated in the fabrication stage. The proposed screen-printed metasurfaces are anticipated to deliver a cost-effective solution capable of propagating MMW radio signals along the length, which can improve the indoor communication to a significant extent.

\section{Design and simulation analysis of the metasurface}

The design and modeling of metasurface as well as the parametric optimisation of the design variables is performed by using the electromagnetic simulation package named CST Microwave Studio 2016, developed on the Finite Integral Techniques (FIT) for EM computations. The design of unitcell at $28 \mathrm{GHz}$ band is initiated with two square patches oriented along the same centre point with a $90^{\circ}$ rotation of the second patch in the same plane. This geometry appeared like an eight (octa)-armed star, which is further modified with a square-shaped slot cut inside, and an outer boundary of parasitic metallic patch lines as shown in Fig. 1 (a). Polyethylene Terephthalate (PET) also known as Melinex, with a thickness of $125 \mu \mathrm{m}$ is selected as a substrate. The unitcell comprises of size $5 \mathrm{~mm}(0.48 \lambda \mathrm{o}) \times 5 \mathrm{~mm}(0.48 \lambda \mathrm{o})$, where $\lambda_{0}$ is the wavelength at $29 \mathrm{GHz}$. The optimised dimensions of the proposed metasurface unit-cell are illustrated in Fig. 1 (b).

The I-shaped metallic resonator structure at $60 \mathrm{GHz}$ in Fig. (c) is designed on two flexible substrates, i.e. Polyamide (Kapton) and Melinex. The thickness of the Melinex $\left(\varepsilon_{\mathrm{r}}=2.9\right.$, $\tan \delta=0.06)$ is $125 \mu \mathrm{m}$, while Kapton $\left(\varepsilon_{r}=3.2, \tan \delta=0.05\right)$ film is $75 \mu \mathrm{m}$ thick. A unit cell of size $1.9 \mathrm{~mm}(0.38 \lambda \mathrm{o}) \times 1.9$ $\mathrm{mm}(0.38 \lambda \mathrm{o})$ with optimised dimensions are shown in Fig. 1 (d), and the designed width of the strip is $0.2 \mathrm{~mm}(0.04 \lambda \mathrm{o})$ where $\lambda_{0}$ is the wavelength at $60 \mathrm{GHz}$. The periodicity of the unit cell is $1.9 \mathrm{~mm}$ while the element spacing was $0.088 \lambda \mathrm{o}$. The geometrical dimensions of the anisotropic metasurface were first tuned to yield a zero reflection phase at $60 \mathrm{GHz}$. The metasurface was designed such that it has a resonant response only in the direction parallel to the $\mathrm{x}$-axis. I-shape was selected due to its intrinsic structural anisotropy in the $\mathrm{x}$-axis, and also maintains a compact size in the orthogonal y-axis [17].

The metasurface is illuminated by a plane wave at a normal incidence angle of the inward travelling plane wave with the electric field polarised in the x-direction. Fig. 2 (a) presents the simulated $S$-parameter results in terms of the reflection (SZmax1Zmax1 and SZmax2Zmax2), and the transmission (SZmin1Zmax1 and SZmin2Zmax2) coefficients and the cross-polarised coupling between the modes. Since both of the designs are symmetrical structures, the co-polar reflection and transmissions of both modes are identical. Fig. 2 (a) show that 


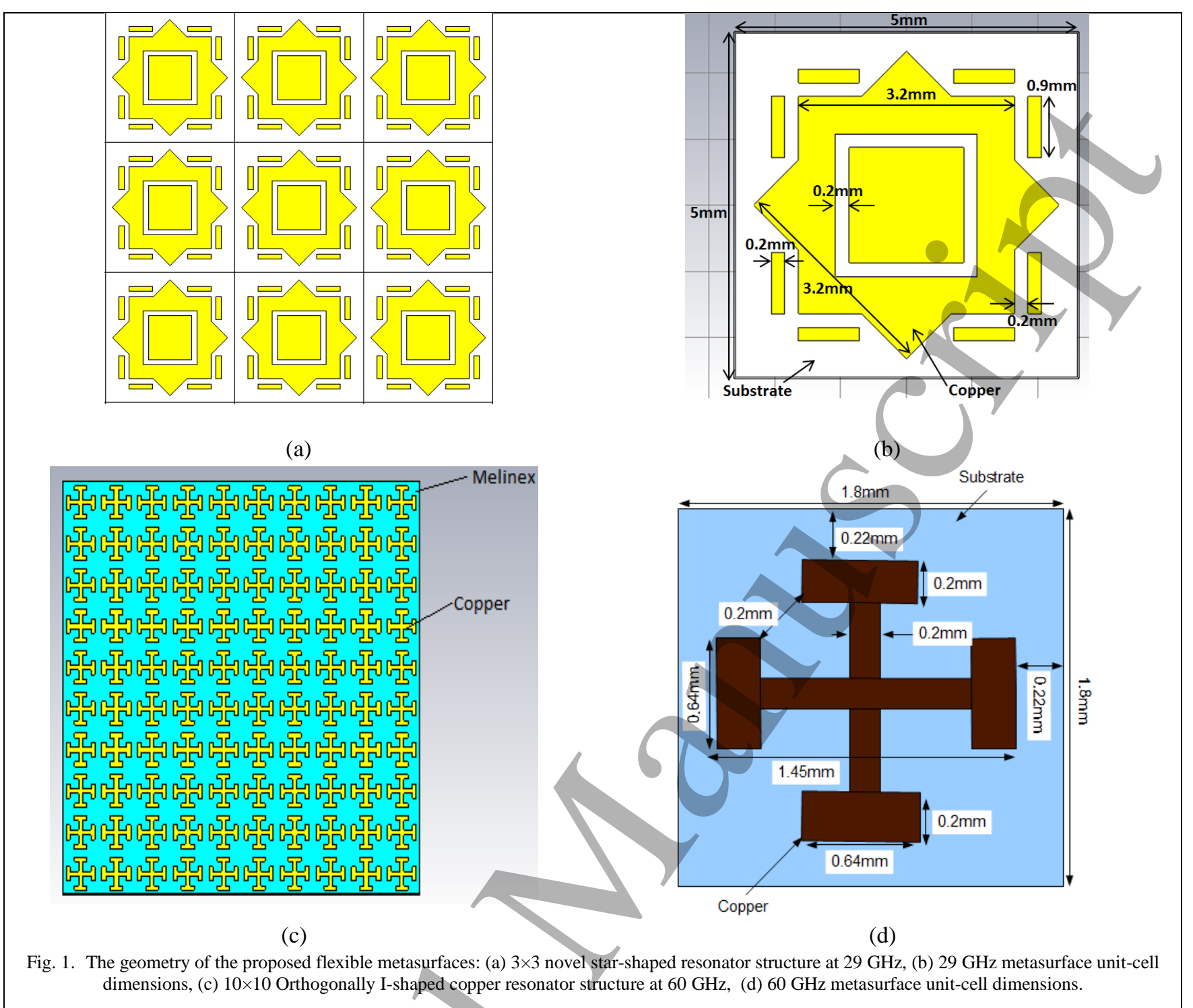

the transmission is almost completely blocked at $28.9 \mathrm{GHz}$ with a magnitude of about $-38 \mathrm{~dB}$ and the reflection is almost complete and has a magnitude of $0.06 \mathrm{~dB}$. A wideband transmission of about $1.5 \mathrm{GHz}(28.1-29.6 \mathrm{GHz})$ has been observed with $-20 \mathrm{~dB}$ as a reference. The reflection phase of the $29 \mathrm{GHz}$ unit cell is shown in Fig. 2 (b) where it coincides with $0^{\circ}$ at the designed frequency of interest.

Similar to the $29 \mathrm{GHz}$ structure, the I-shaped metasurface at $60 \mathrm{GHz}$ is also illuminated by a plane wave at a normal incidence angle with the E-field polarised along the $\mathrm{x}$-axis. The simulated results of Fig. 3 (a) present that the transmission is almost completely blocked at $60 \mathrm{GHz}$ as the plot shows a magnitude of $-43 \mathrm{~dB}$ and the reflection is almost complete with $0.04 \mathrm{~dB}$ magnitude. A wideband transmission has been observed of $3.5 \mathrm{GHz}(58.25-61.75 \mathrm{GHz})$. The cross-polarised coupling between the modes is below $-70 \mathrm{~dB}$. The reflection phase of the unit cell coincides with $0^{\circ}$ at $60 \mathrm{GHz}$ as shown in Fig. 3 (b). The transmission coefficient has a phase coverage of $22 \pi\left( \pm 180^{\circ}\right)$ as expected for such metasurface structures.

\section{Principle of operation}

In this section, the principle of operation has been explained only for the $60 \mathrm{GHz}$ metasurface as the $29 \mathrm{GHz}$ structure operates in a similar way. To limit the computational time of the modeling, the metasurface array size was reduced to $5 \times 10$ unit-cells, with a total size of $18 \mathrm{~mm}(3.6 \lambda \mathrm{o}) \times 9 \mathrm{~mm}$ (1.8 $\lambda$ o). Fig. 4 (a) shows a rectangular waveguide tapered at the edge for an effective passage of the transmitted signal by means of the metasurface structure placed next to it. Since waveguides transmit EM signals at a lower loss than coaxial cables [26], the same phenomenon is inculcated in this work with a minimum cross-section, relative to the signal wavelength in order to function properly. For this purpose, a rectangular waveguide-WR15 (WG25) that cover $50-75 \mathrm{GHz}$ with size $3.76 \mathrm{~mm} \times 1.88 \mathrm{~mm}$ is used [27]. The numerical simulation of the electric field distribution is shown in Fig. 4 (b) and (c). The EM-waves' manipulation at a subwavelength 


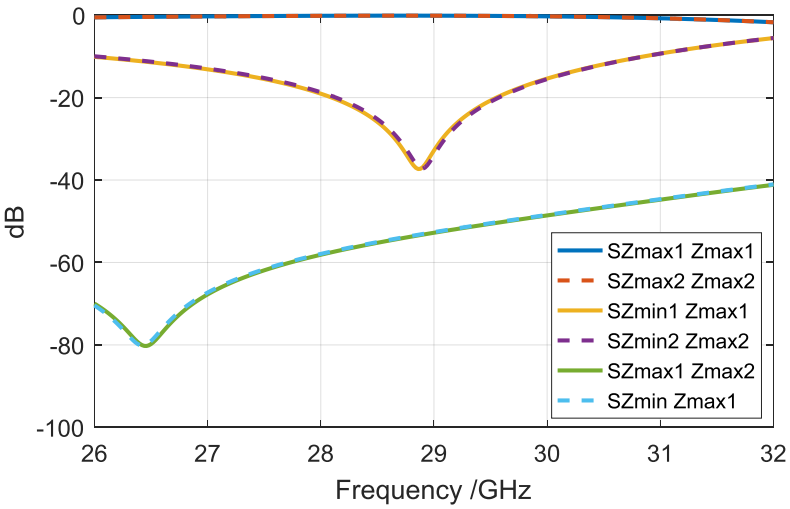

(a)

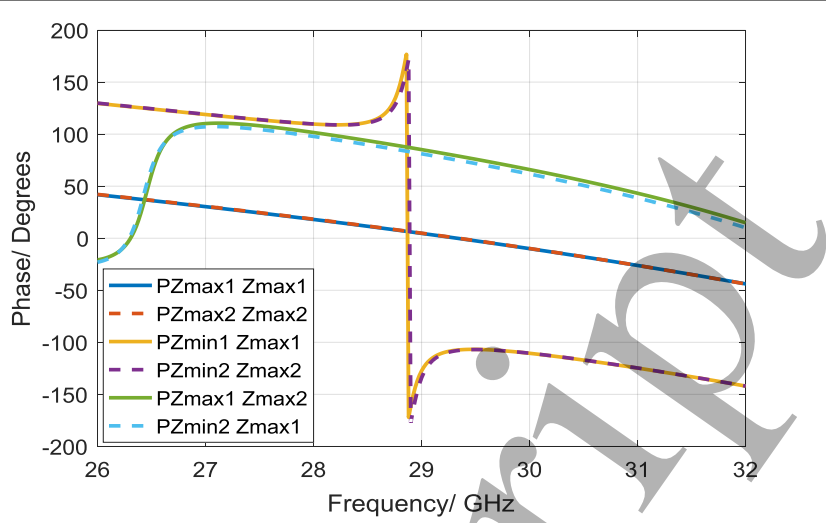

(b)

Fig. 2. Magnitude and phase of the $29 \mathrm{GHz}$ metasurface. (a) S-parameters of the reflection coefficient, transmission coefficient and cross-polarized coupling being mode, (b) Phase of the reflection coefficient, transmission coefficient and cross-polarized coupling being mode.

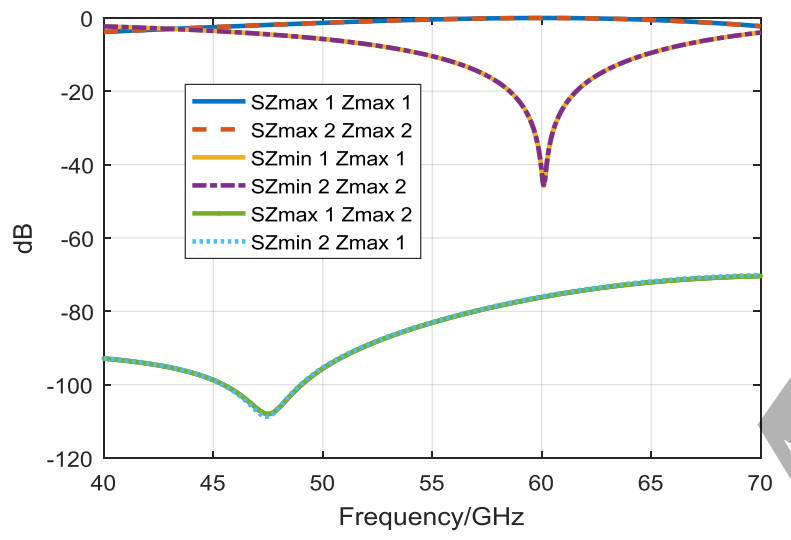

(a)

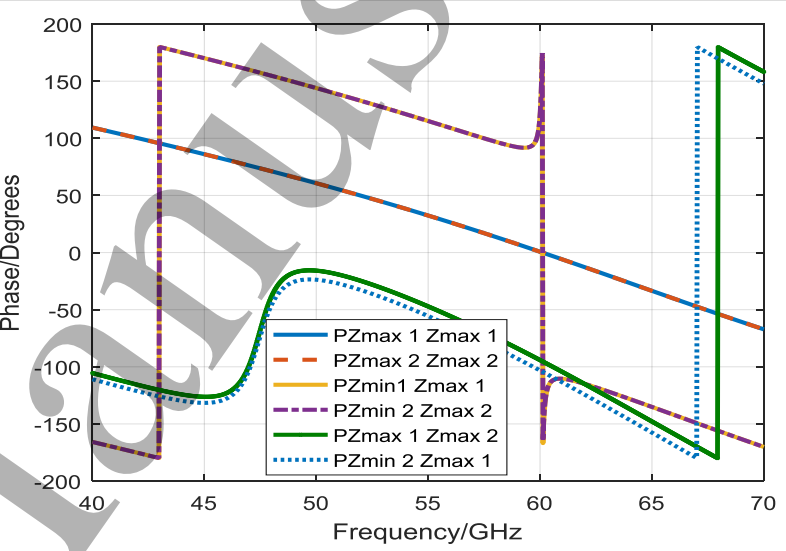

(b)

Fig. 3. Magnitude and phase of the $60 \mathrm{GHz}$ metasurface. (a) S-parameters of the reflection coefficient, transmission coefficient and cross-polarized coupling being mode, (b) Phase of the reflection coefficient, transmission coefficient and cross-polarized coupling being mode

level can be achieved by patterning the metallic surface of the waveguide directly to the metasurface structure. The surface radiates a highly collimated beam, whose divergence indicates a good waveguiding capability of the metasurface medium. The designed metasurface is used in this research as a bandpass filter at the desired frequency band.

It can be anticipated that metasurface is less absorbent than the 3D metamaterial structures, thus the signal transmission by means of electric field through the metasurface becomes easier over a long distance [28]. It is observed that at $50 \mathrm{GHz}$, the EM waves transmission across the metasurface shows lesser signal strength over the whole surface as in Fig. 4 (b), while at $60 \mathrm{GHz}$ it performs like a pass band for the signal as in Fig. 4 (c). It is worth mentioning that the orthogonal Ishaped structure exhibits inductive and capacitive reactance by the excitation of the electric field. The inductance occurs as a result of the straight I-shaped conductors and the capacitance is due to the capacitive coupling between adjacent I-shaped structures [29].

\section{Fabrication of the designed flexible metasurface}

The realisation of the designed MMW metasurfaces on flexible films involves several critical challenges due to the factors which affect the reliability, robustness and accuracy of the printed electronics. Selection of a suitable process which can ensure precise fabrication of the small-scale dimensions and also delivers time-efficient and cost-effective production is highly desired. Choice of a flexible substrate is another important aspect among the commercially available options. Melinex ST 504 is the widely available thermoplastic polymer from the polyester family. Because of its recycling capability, low cost and eco-friendly attributes, it is manufactured in bulks and has been made part of clothing, disposable packing materials and thermoforming, and also in the manufacture of several resins when blended with glass fibre [30]. Thin sheets of Melinex/PET, varying in composition, permittivity and film thickness have been utilised in flexible electronics [31-33]. Besides, polyimide film developed by DuPont (Kapton), has 


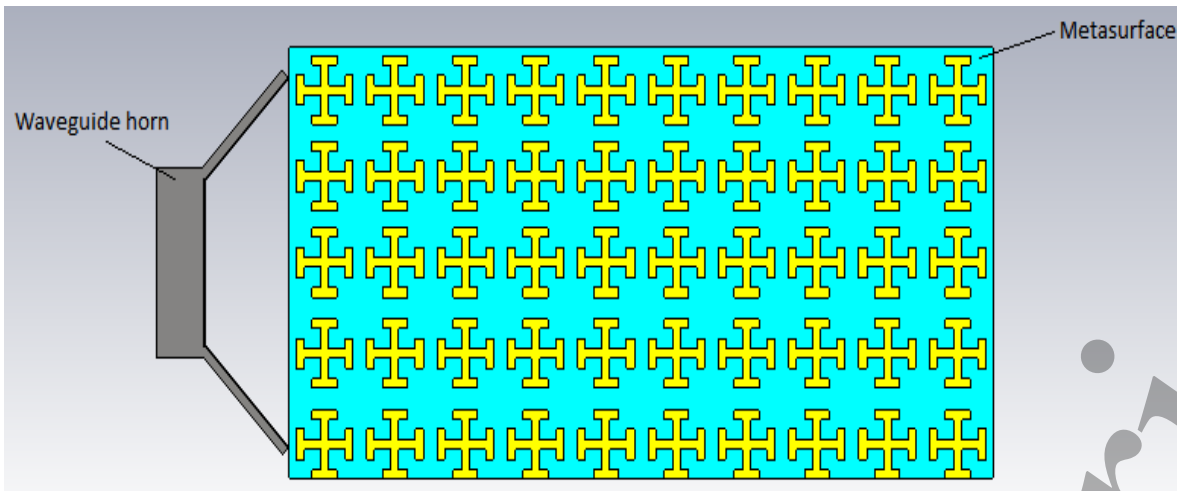

(a)

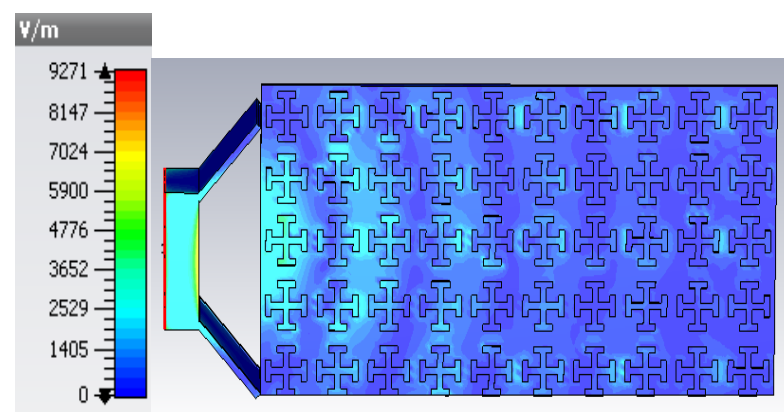

(b)

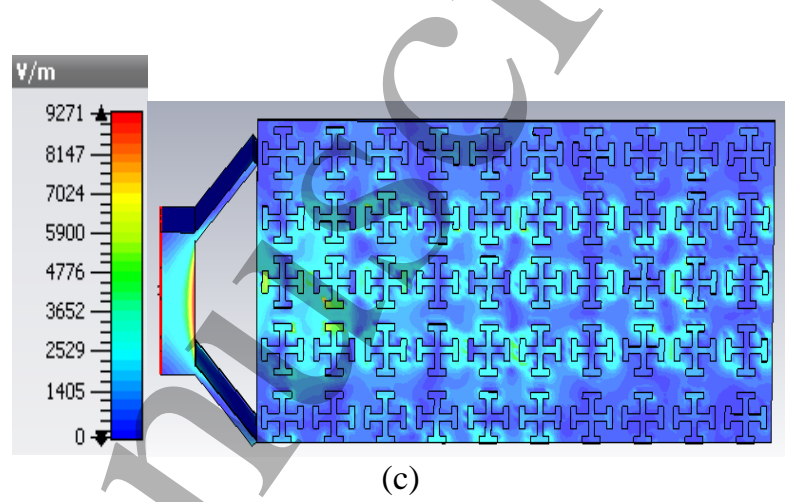

Fig. 4. The electric field of $5 \times 10$ orthogonally I-shaped resonator metasurface with a rectangular waveguide: (a) top view (b) Electric field distribution on the metasurface at $50 \mathrm{GHz}$ and (c) Electric field distribution on the metasurface at $60 \mathrm{GHz}$.

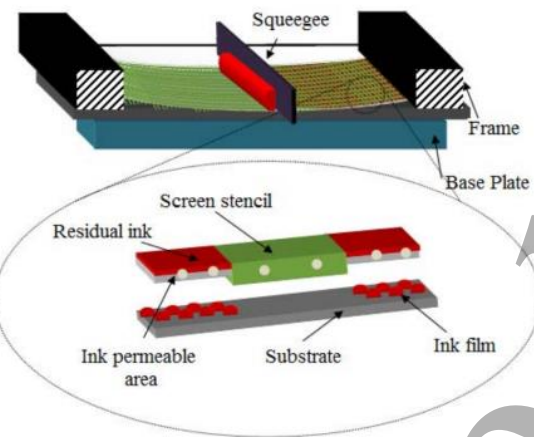

(a)

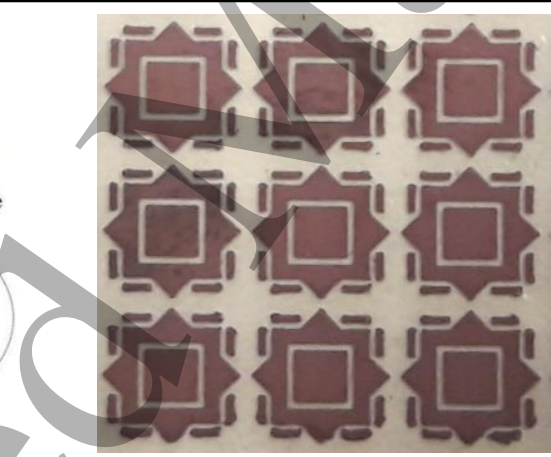

(b)

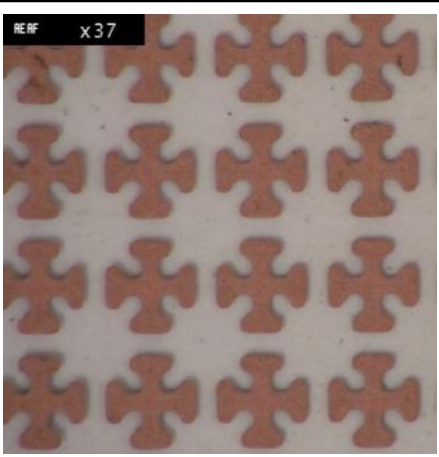

(c)

Fig. 5. The fabricated prototypes of the metasurfaces by screen printing process: (a) representation of screen printing process [39], (b) 29 GHz screen-printed metasurface, (c) $60 \mathrm{GHz}$ screen-printed metasurface.

excellent properties of flexibility, mechanical, chemical and thermal stability, inert and highly hydrophobic surfaces etc. and widely used for flexible electronics [34]. Melinex and Kapton sheets have been used as the substrate in this research work due to their flexibility and durability.

Several methodologies have been developed to accomplish the fabrication of flexible films. Flexible substrates added with a metal such as copper or aluminium can be laser etched, yet requires an expensive laser milling equipment [35]. Inkjet printing is an additive manufacturing technique for precise fabrication of flexible films by using an inkjet printer [36]. This approach has been successfully extended to a reel-to-reel process for a mass production. Photolithography is a process of transferring a pattern on a substrate by means of light and involves highly sophisticated clean-room facilities to achieve high accuracy in prototyping [37]. Another feasible method which avoids the high cost of machinery required for laser etching, inkjet printing or cleanroom facilities and delivers reasonable fabrication accuracy is the screen printing process [38]. The screen printing involves the use of an ink-blocking stencil that defines the area to be patterned on the substrate by means of a conductive paste. In this research, the screen printing process has been selected due to the low cost of the equipment, good printing quality with high conductivity, and

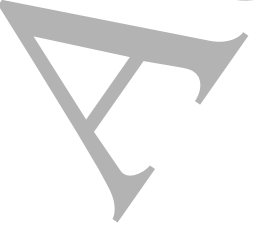




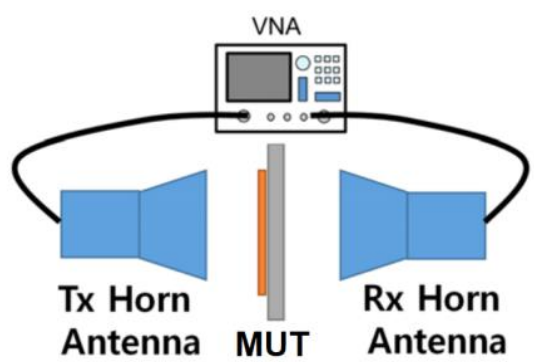

(a)

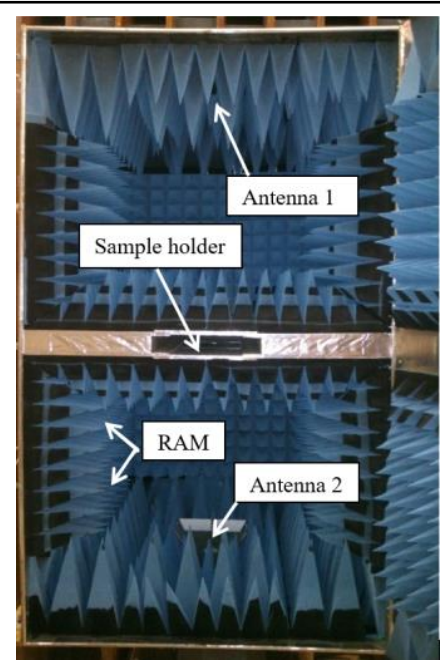

(b)

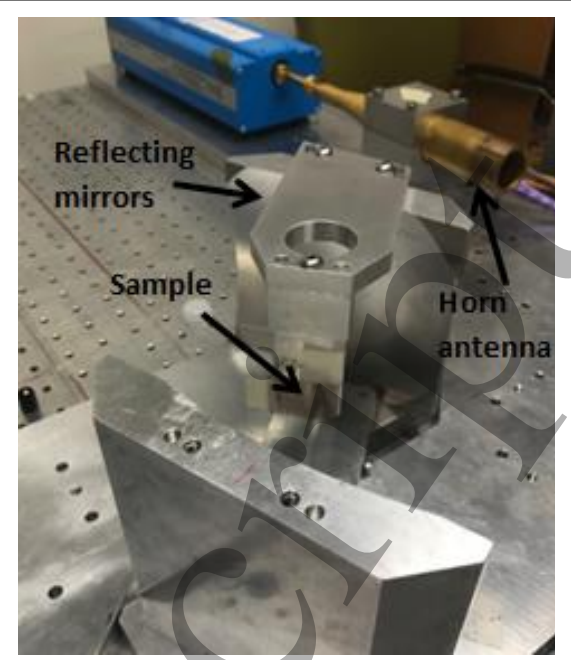

(c)

Fig. 6. Testing of transmission coefficient of the designed metasurfaces: (a) Operational principle of the measurements [40], (b) Setup for measurement of transmission at $28 \mathrm{GHz}$, (c) Quasi-optic bench for the measurement of transmission at $60 \mathrm{GHz}$.

reasonable accuracy of the printed pattern. The proposed metasurfaces at $29 \mathrm{GHz}$ and $60 \mathrm{GHz}$ are fabricated using the screen printing followed by a sintering process.

A number of choices are available in the market regarding the conductive inks, pastes and epoxies. Intrinsiq UK suppies different variants of the paste based on varying the composition, wettability, adhesion, and conductivity, mainly dependent on the concentration of the copper content in the solvent. Nanoparticle copper paste CP-005 with a solid weight of $\sim 85 \%$, viscosity of $15-25 \mathrm{~Pa} . \mathrm{S}$, density of $\sim 3.5 \mathrm{~g} / \mathrm{ml}$, from the Intrinsiq UK is selected. Fig. 5 (a) presents the screen printing process to transfer the pattern from screen to the substrate. The screen is made with a fine stainless steel mesh coated with a $12 \mu \mathrm{m}$ thick photoemulsion with an engraved pattern to transfer the paste. For the screen printing, a 29-inch screen with a $45^{\circ}$ tilt is deployed, designed with a standard mesh count of 325/inch and a wet print thickness of $\sim 62 \mu \mathrm{m}$. The approximate aperture size is $42 \mu \mathrm{m}$ and mesh opening ratio is $\sim 39 \%$. A reasonable level of accuracy can be achieved by a careful deployment. The next step involves the sintering of the printed metasurface which is performed in the manufacturing facilities of Intrinsiq UK. The laser beam is exposed on the printed surface by using formic acid in a nitrogen atmosphere. Belt furnace with formic acid dosing system is used where laser sintering is carried out on the FASTLAPS roll-fed prototype system developed from the funding of Innovate UK. The desired level of conductivity is achieved after the sintering process of the printed prototype. The thickness of the printed copper film is approximately 7 $10 \mu \mathrm{m}$ and sheet resistance computed by using a 2-point probe test range from $0.03-0.07 \Omega$ depending on the temperature as well as different variants of copper paste being used for the analysis. These values suggest that the computed conductivity of the printed sheet is approximately $1.4 \times 10^{6}-3.3 \times 10^{6} \mathrm{~S} / \mathrm{m}$. The fabricated prototypes of the designed metasurfaces at 29
GHz and $60 \mathrm{GHz}$ are shown in the Fig. 5 (b) and (c). The adhesion quality of the printed prototypes is tested by bending and folding, as well as by scotch tape test. These destructive testing techniques have ensured highly reliable performance of the screen printed patterns in strain conditions as no visible cracks or damage is observed.

\section{The experimental results and analysis}

Fig. 6(a) shows the operating principle of the experimental setup for the transmission characteristics of the metasurface, with an orthogonally orientated sample placed between the two antennas. Here, a sample (metasurface under test-MUT) is placed in between two horn antennas and a vector network analyzer (VNA) is used to measure the $S$-parameters. In order to perform these measurements, separate setups have been developed for each metasurface. For instance, the metasurface at $29 \mathrm{GHz}$ is tested in the characterisation chamber, which consists of a setup with a pair of $\mathrm{K}_{\mathrm{a}}$-band horn antennas as shown in Fig. 6 (b). The sample is placed between the horn antennas at the specified distance, while the transmission characteristics are obtained from the PNA-X. In addition, the Kapton and Melinex based metasurfaces designed at $60 \mathrm{GHz}$ are tested by using a quasi-optic bench, which comprises of an arrangement of corrugated horns designed for the frequency range of 50-75 GHz, reflecting mirrors, and a sample holder where beams of the antennas converge to transmit through the sample. Fig. 6 (c) presents a quasi-optic bench for the testing of $60 \mathrm{GHz}$ metasurface.

Multiple samples taken from the extended metasurfaces printed with the CP-005 copper-paste are examined in this stage. The measurements presented in Fig. 7 (a) show that the designed metasurface has an operating bandwidth of 26-31 GHz. The accuracy and reliability of the results is determined by the repeatabity of measurements of multiple samples taken 


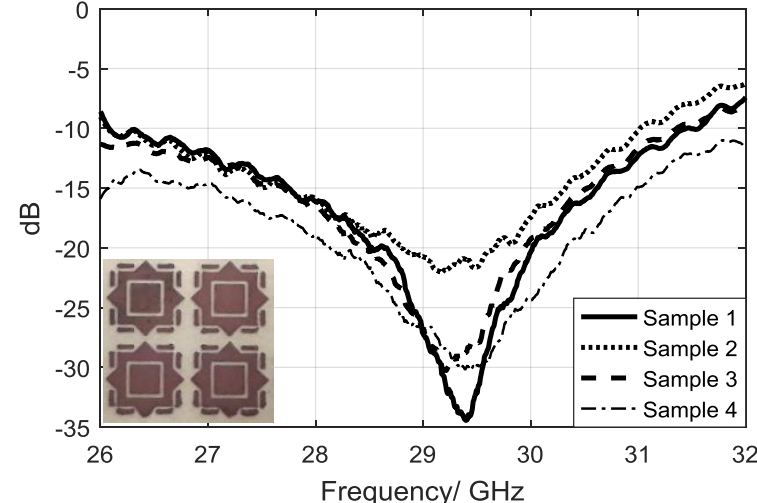

(a)

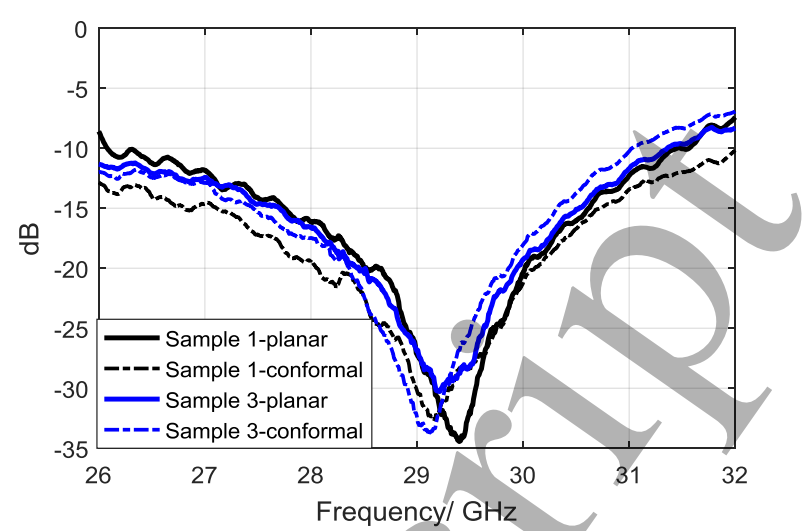

(b)

Fig. 7. Measurements of transmission characteristics of the Melinex based octa-armed star-shaped metasurface at 29 GHz: (a) measured plots of printed samples cut from the extended metasurface, (b) Comformal analaysis of the two samples when placed on a planar and conformal mount.

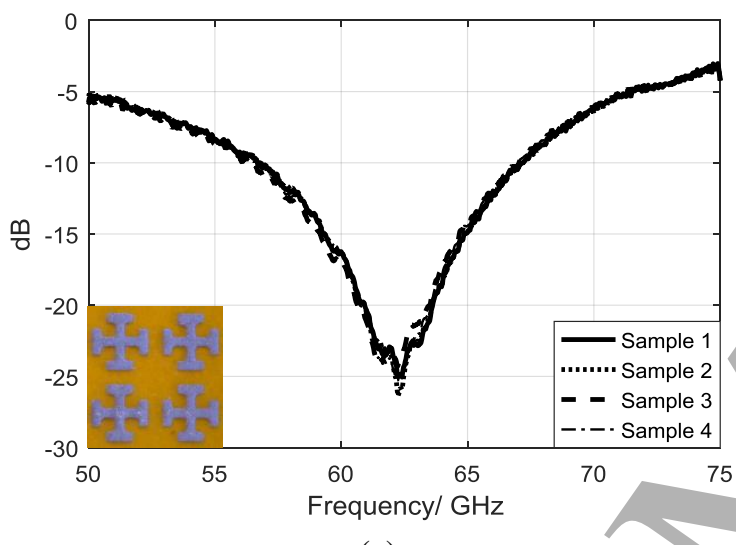

(a)

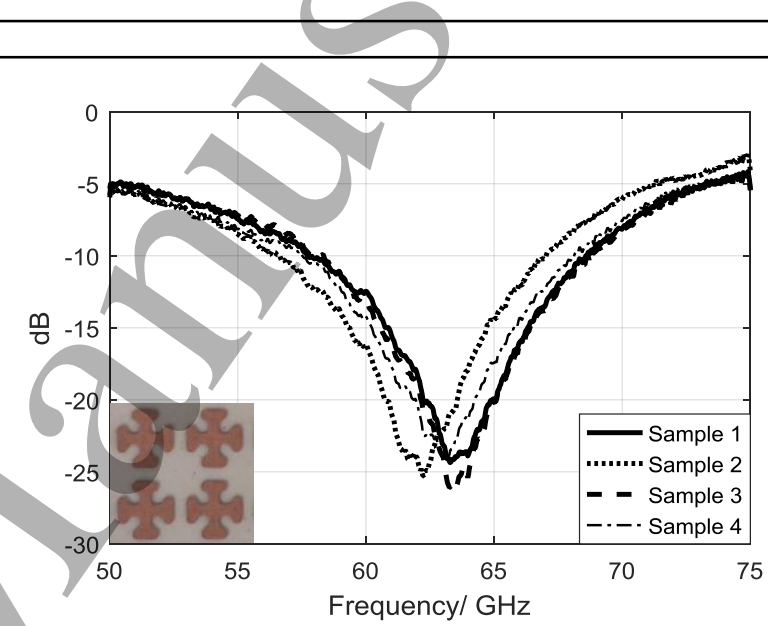

(b)

Fig. 8. Measurements of transmission characteristics of the designed I-shaped metasurface at $60 \mathrm{GHz}$ : (a) Samples of metasurface fabricated on Kapton film, (b) Samples of metasuface fabricated on Melinex substrate.

along the long metasurface film. Samples from various parts of the film have been tested which show that the bandwidth remains conserved with $-10 \mathrm{~dB}$ reference, though have some variations in the magnitudes at the resonant dips as shown in Fig. 7 (a). In order to investigate the performance in conformal integrations, a conformal surface of polystyrene is used to hold the sample and the measurements are compared with the flatbed results in Fig. 7 (b). Minor shifts of resonant frequency have been observed, however, the overall operating bandwidth is conserved to ensure a consistent performance. The $60 \mathrm{GHz}$ metasurface samples printed on Kapton and PET substrates show a sharp dip between $60-65 \mathrm{GHz}$ in Fig. 8. Also, the measured bandwidth of the Kapton metasurface is 57.3-67.5 $\mathrm{GHz}$ and that of Melinex is approximately $57.5-67.5 \mathrm{GHz}$ as shown in Fig. 8 (a) and (b) respectively. It is observed that the Kapton based samples show negligible variations and converged plots as compared to the Melinex samples, which establishes a highly stable performance at high frequencies.
Instead, the slight shift observed in the case of Melinex samples' measurement plots for both $60 \mathrm{GHz}$ and $29 \mathrm{GHz}$ could be due to small variations in the dielectric constant of the Melinex at higher frequencies, however, Kapton shows high stability in this regard.

\section{Conclusion}

This paper presents the concept of utilizing metasurfaces effectively in order to manipulate the EM performance by using the state-of-the-art testing facilities. Two distinctive bands of MMW spectrum i.e. $29 \mathrm{GHz}$ and $60 \mathrm{GHz}$ have been used to investigate the performance of designed metasurfaces. The flexible metasurface has been fabricated by the process of screen printing with conductive nanoparticle copper ink/paste. The metasurface has been designed to interact with both electric and magnetic field component of EM wave, thus made this to successfully manipulate wavefront and maximise power transmission or reflection simultaneously. A good

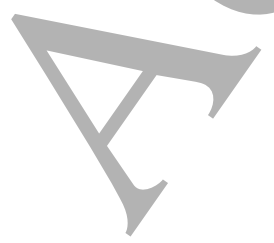


agreement has been observed between the simulated and measured results. The proposed metasurface at $29 \mathrm{GHz}$ has achieved a wide transmission bandwidth of $26-31 \mathrm{GHz}$, and $60 \mathrm{GHz}$ metasurface offers $57.5-67.5 \mathrm{GHz}$ bandwidth, with the efficiency of $80 \%$ and above at these frequencies of consideration. The flexiblity and conformity of the proposed metasurfaces have made the proposed metasurfaces capable to integrate into a non-planar environment. From the results, it is anticipated that the metasurface could serve to enhance the strength of the signals propagated directly into buildings (indoors) for delivering high-speed broadband as a profitable replacement of copper/optical fibre in near future.

\section{Acknowledgement}

The authors gratefully acknowledge the grants 132216 AMMETEX - Advanced Materials and Metamaterial Structures for MetaTextiles, and EP/N029666/1 Enabling High-Speed Microwave and Millimetre Wave Links (MiMiWaveS) for providing foundational support that made this research possible.

\section{References}

[1] Chang J S, Facchetti A F, and Reuss R 2017 A circuits and systems perspective of organic/printed electronics: review, challenges, and contemporary and emerging design approaches IEEE J. Emerg. Sel. Topics Circuits and Syst., 7 1 7-26

[2] Mashayekhi M et al 2016 Evaluation of aerosol, superfine inkjet, and photolithography printing techniques for metallization of application specific printed electronic circuits IEEE Trans. Electron Devices 633 1246-1253

[3] Olkkonen J, Lehtinen K, and Erho T 2010 Flexographically printed fluidic structures in the paper Anal. Chem. 822410246 10250

[4] Wei Y et al 2013 Screen printing of a capacitive cantilever-based motion sensor on fabric using a novel sacrificial layer process for smart fabric applications Meas. Sci. Technol. 247075104

[5] Watson D E, Ng J H, and Desmulliez M P Y 2011 Additive photolithography based process for metal patterning using chemical reduction on surface modified polyimide 18th European Microelectronics \& Packaging Conf. 1-7.

[6] Khan S, Lorenzelli L, and Dahiya R S 2015 Technologies for printing sensors and electronics over large flexible substrates: A review IEEE Sensors J. $1563164-3185$

[7] Jilani S F et al 2018 Low-profile flexible frequencyreconfigurable millimetre-wave antenna for $5 \mathrm{G}$ applications Flex. Print. Electron., 3035003

[8] Veselago V 1968 The electrodynamics of substances with simultaneously negative values of $\varepsilon$ and $\mu$. Sov. Phys. Uspekhi 10 509-514

[9] Pendry J B et al 2012 Transformation optics and subwavelength control of light Science 3376094 549-552

[10] Li Y et al 2014 Experimental realization of full control of reflected waves with subwavelength acoustic metasurfaces Phys. Rev. Appl. 26064002

[11] Guenneau S et al 2007 Acousticmetamaterials for sound focusing and confinement New J. Phys. 9399
[12] Qu C, Zhu C, and Kinzel E C 2018 Fabrication of infrared broadband polarized emitting metasurfaces using microsphere photolithography Proc. SPIE 10544, Adv. Fabrication Technol. for Micro/Nano Optics and Photonics XI, 105440C

[13] Zhao Y, Liu X X, and A. Alu A 2014 Recent advances on optical metasurfaces," Journal of Optics, 1612 1-14, 123001

[14] Chen H T, Taylor A J, and Yu N 2016 A review of metasurfaces: physics and applications Rep. Prog. Phys. 79 076401

[15] $\mathrm{Yu} \mathrm{N}$, and Capasso F 2014 Flat optics with designer metasurfaces Nat. Mater. 13 139-150

[16] Buchnev O et al 2015 Electrically controlled nanostructured metasurface loaded with liquid crystal: toward multifunctional photonic switch Adv.Opt. Mater. 3 674-679

[17] Lin J et al 2013 Polarization-controlled tunable directional coupling of surface plasmon polaritons Science 340 331-334

[18] Aieta F et al 2012 Aberration-free ultrathin flat lenses and axicons at telecom wavelengths based on plasmonic metasurfaces Nano Lett. $1294932-4936$

[19] Yin X et al 2013 Photonic spin hall effect at metasurfaces Science 339 1405-1407

[20] Sun S et al 2012 Gradient-index meta-surfaces as a bridge linking propagating waves and surface waves Nat. Matter. 11 426-431

[21] Rappaport T S et al 2017 Overview of millimeter wave communications for fifth-generation $(5 \mathrm{G})$ wireless networkswith a focus on propagation models IEEE Trans. Antennas Propag. 6512 6213-6230

[22] Rappaport' T S et al 2013 Millimeter-wave mobile communications for $5 \mathrm{G}$ cellular: It will work! IEEE Access $\mathbf{1}$ 335-349

[23] Liu P, Renzo M D, and Springer A 2016 Line-of-sight spatial modulation for indoor mmWave communication at $60 \mathrm{GHz}$ IEEE Trans. Wireless Commun. $15117373-7389$

[24] A. Rahimian A, Alfadhl Y, and Alomainy A 2016 Analytical and numerical evaluations of flexible V-band Rotman lens beamforming network performance for conformal wireless subsystems Progress In Electromagnetics Research B 71 77-89

[25] Wang L B et al 2011 Ultrathin and flexible screen-printed metasurfaces for EMI shielding applications IEEE Trans. Electromagn. Compat. 53 300-705

[26] Khan A S, Waveguides Microwave Engineering: Concepts and Fundamentals (CRC Press Taylor \& Francis Group, Boca Raton) p. 129

[27] Cross reference for hollow metallic waveguides Technical information, Spinner TD-00036 pp. 1-13, 2014. www.spinnergroup.com

[28] Zhu A Y et al 2016 Traditional and emerging materials for optical metasurfaces Nanophotonics 62 452-471

[29] Langley R J, and Drinkwater A J 1982 Improved empirical model for the Jerusalem cross IEE Microw Opt. Antennas 1291 $1-6$

[30] Jilani S F, and A. Alomainy A 2016 Planar millimeter-wave antenna on low-cost flexible PET substrate for 5G applications 10th European Conf. Antennas and Propag. (EuCAP) 1-3

[31] Guo X et al 2017 Flexible and wearable $2.45 \mathrm{GHz}$ CPW-fed antenna using inkjet-printing of silver nanoparticles on PET substrate Microw. Optical Tech. Lett. 591 204-208 
[32] Jilani S F and Alomainy A 2017 An inkjet-printed MMW frequency-reconfigurable antenna on a flexible PET substrate for 5G wireless systems Loughborough Antennas Propag. Conf. (LAPC) 1-3

[33] Na K et al 2016 Graphene-based wireless environmental gas sensor on PET substrate IEEE Sensors J. 1612 5003-5009

[34] Fang Y et al 2016 A bio-enabled maximally mild layer-bylayer Kapton surface modification approach for the fabrication of all-inkjet-printed flexible electronic devices Sci. Rep. 6, 39909

[35] Rabbani M S, and Ghafouri-Shiraz H 2017 Liquid crystalline polymer substrate-based $\mathrm{THz}$ microstrip antenna arrays for medical applications IEEE Antennas Wireless Propag. Lett. 16 $1533-1536$

[36] Sridhar A et al 2009 Inkjet-printing- and electroless-platingbased fabrication of RF circuit structures on high-frequency substrates J. Micromech. Microeng. 19085020

[37] Toriz-Garcia J J et al 2013 Fabrication of a 3D electrically small antenna using holographic photolithography J. Micromech. Microeng. 23055010

[38] Xiao G et al 2018 Printed UHF RFID reader antennas for potential retail applications IEEE Journal of Radio Frequency Identification 2 1 31-37

[39] Eshkeiti A et al 2015 Screen printing of multilayered hybrid printed circuit boards on different substrates IEEE Trans. Compon., Packag., Manuf. Technol. 5 3 415-421

[40] Malik J el al 2018 Electromagnetically induced transparency in sinusoidal modulated ring resonator Appl. Phys. Lett. 11234102 